\title{
INTERNAL FIXATORS: A SAFE OPTION FOR MANAGING DISTAL FEMUR FRACTURES?
}

\author{
Bruno Bellaguarda Batista ${ }^{1}$, Rodrigo Salim ${ }^{1}$, Cleber antonio Jansen Paccola ${ }^{2}$, Mauricio Kfuri Junior ${ }^{1}$
}

\begin{abstract}
Objective: Evaluate safety and reliability of internal fixator for the treatment of intra-articular and periarticular distal femur fractures. Methods: Retrospective data evaluation of 28 patients with 29 fractures fixed with internal fixator was performed. There was a predominance of male patients (53.5\%), with $52 \%$ of open wound fractures, $76 \%$ of $\mathrm{AO} 33 \mathrm{C}$ type fractures, and a mean follow up of 21.3 months. Time of fracture healing, mechanical axis deviation, rate of infection and postoperative complications were registered. Results: Healing rate was $93 \%$ in this sample, with an average time of 5.5 months. Twenty-seven percent of patients ended up with mechanical axis deviation, mostly resulting from poor pri-
\end{abstract}

mary intra-operative reduction. There were two cases of implant loosening; two implant breakage, and three patients presented stiff knee. No case of infection was observed. Healing rate in this study was comparable with current literature; there was a high degree of angular deviation, especially in the coronal plane. Conclusion: Internal fixators are a breakthrough in the treatment of knee fractures, but its use does not preclude application of principles of anatomical articular reduction and mechanical axis restoration. Level of Evidence II, Retrospective Study.

Keywords: Femur. Internal fixators. Bone plates.

Citation: Batista BB, Salim R, Paccola CAJ, Kfuri Junior M. Internal fixators: a safe option for managing distal femur fractures? Acta Ortop Bras. [online]. 2014;22(3):159-62. Available from URL: http://www.scielo.br/aob.

\section{INTRODUCTION}

Distal femur fractures represents about $7 \%$ of all femur fractures in adults, and are notoriously difficult to treat. ${ }^{1}$ These lesions have a bimodal distribution affecting both elderly patients, mostly women with osteoporotic bone, victims of low-energy trauma, and young patients involved in automobile accidents, high energy trauma, in this case polytrauma with complex intra-articular often associated with open wound fractures. ${ }^{2}$

Historical treatment of these fractures can be divided into two distinct periods. Until the late 1960s, most authors advocated conservative treatment of such injuries with traction and cast immobilization. At that time surgical treatment was still discouraging because of high rate of complications. ${ }^{3,4}$

Reporting of good results with the surgical techniques recommended by the $\mathrm{AO}$ group (Arbeitsgemeinschaft für Ostheosynthesefragen) since the early 1970s, built up a new perspective on therapeutic approach to distal femur fractures, prioritizing anatomical reduction, fracture stabilization and early knee mobilization. ${ }^{5}$

Implant developments experienced in recent decades, namely the launching of locking plates has been associated with new possibilities in fracture treatment. ${ }^{2,6-9}$ The shift from mechanical approaches to biological ones has also brought a different percepcion in the management of distal femur fractures. This "biological" approach to fracture is considered to cause lower rates of complications. ${ }^{7,9,10}$ Numerous techniques are described for surgical treatment of distal femur fractures. They may be extramedullarly, with the aid of plates and screws, or intramedullary. Factors that guide this choice are related to fracture pattern, bone quality, functional demands and type of trauma. Because of challengeable fixation in much comminuted intra-articular fractures with very short epiphyseal fragment in osteoporotic bone, a new generation of implants has been developed. These are the internal fixators, plates on which screw head is locked to implant hole, providing angular stability to the assembly. These new plates act biomechanically as external fixator, with biomechanical stability screw-hole similar to externar fixator connector-tube unit. ${ }^{11}$

Best known internal fixator system for distal femur is LISS $^{\circledR}$ (Less Invasive Stabilization System - Synthes), which has the option to be introduced percutaneously, by minimally invasive techniques. This plate was designed according to distal femur

All the authors declare that there is no potential conflict of interest referring to this article.

\footnotetext{
1. Faculdade de Medicina de Ribeirão Preto, Universidade de São Paulo, Ribeirão Preto, SP, Brazil.

2. (In memoriam), Faculdade de Medicina de Ribeirão Preto, Universidade de São Paulo, Ribeirão Preto, SP, Brazil.

Work performed at the Department de Biomechanics, Medicine and Rehabilitation of the Locomotor Apparatus - Faculdade de Medicina de Ribeirão Preto da USP, Ribeirão Preto, SP, Brazil.

Correspondence: Mauricio Kfuri Junior, Departamento de Biomecânica, Medicina e Reabilitacão do Aparelho Locomotor, Faculdade de Medicina de Ribeirão Preto (USP) 14048-900
} Ribeirão Preto, SP, Brazil. kfuri@fmrp.usp.br; brunobellaguarda@yahoo.com.br 
anatomy, requiring no additional modeling.$^{11}$ Furthermore, biomechanical testing showed better control of angular deviation compared to the conventional plates. ${ }^{12}$ Although internal fixators reflect a potential breakthrough in the treatment of fractures in osteoporotic bone and in that cases where epiphyseal segment is short, they have as main disadvantage their high cost, making them less accessible to most orthopedic services in developing countries. As every new tool, those plates were understood as the ultimate solution for all problems related to distal femur fractures management.

The aim of this study was to evaluate safety and reliability of internal fixators (LISS ${ }^{\circledR}$ ) for articular and periarticular distal femur fractures treatment (33-A and 33-C).

\section{MATERIALS AND METHODS}

Patients with distal femur fractures (AO 33-A or 33-C) treated with LISS ${ }^{\circledR}$ plate from April 2002 to December 2008 were included in this study. This retrospective study was approved by the ethics committee of the University Hospital of Ribeirão Preto-USP, all patients agreed to participate to this research. All patients were operated by the same trauma team, comprised of two faculty members and three medical assistants with experience in this type of injury. Patients underwent the same treatment protocol.

Definitive treatment included direct reduction of articular fractures an indirect reduction of metaphyseal fragments. The patient was positioned in an ordinary radiolucent table in supine position. Prophylactic antibiotic was administered approximately 30 min before the beginning of the procedure, and medication was kept for at least 24 hours postoperatively. Two options of incisions were used based on the fracture pattern: lateral parapatellar incision for articular fractures; or in cases of metaphyseal without articular fracture was used a lateral incision. Articular reduction was achieved by use of bone reduction forceps, and Kirschner wire (KW) temporarily, than fixed with $6.5 \mathrm{~mm}$ cancellous screw with washer. After attaining the reduction the plate was percutaneously placed (MIPO), and fixed to the bone. Reestablishment of length and axis of the femur was done manually or using an AO fracture distractor and KW as "joystick". After definitive surgery, patient was kept for at least 48 hours in hospital, where physiotherapy was initiated without load, and early mobilization of the knee. Ten patients required prolonged hospitalization for enhancing physiotherapy program with continuous passive motion machine (Continuous Passive Motion - CPM) due to difficulty in gaining knee mobility. Those cases were associated with more complex fracture and soft tissue injury patterns.

Clinical follow-up was performed at the first visit one week postoperatively for wound inspection. The second visit, eight weeks after surgery, included X-ray control in addition to clinical evaluation. The number of visits depended on the patient's clinical condition, but all of them were evaluated in a standardized manner at 6 and 12 months postoperatively.

Imaging study allowed characterization of fracture patterns according to the $\mathrm{AO}$ classification, healing time and measurement of the anatomical axis of the femur in both sagittal and coronal plans, right after operation and after complete fracture consolidation. An $81^{\circ}$ anatomical axis in the frontal plan was considered normal. In the sagittal plane, following population averages shown in the literature, deviations of up to $10^{\circ}$ were considered tolerable. ${ }^{13}$

All medical procedures to deal with any complications related to fracture and its fixation were properly recorded.

\section{RESULTS}

Thirty-two patients with distal femoral fractures were operated in this service during the study period. Four did not complete six months of follow-up and were excluded from this study. Among the remaining 28 patients, 15 were male $(53.5 \%)$ and 13 were female (46.5\%) with mean age of 47.3 years old.

Twenty-nine fractures were treated with LISS $^{\circledR}$ plate, 18 left femur (62\%) and 11 right femur(38\%), with a mean follow up of 21.3 months (range 12-66). According to the AO classification, 24\% were type 33A (two 33-A1; one 33-A2; and four 33-A3), and 76\% were type 33C (three 33-C3, seven 33-C2; and twelve 33-C3). High-energy trauma mechanism was predominant with $82 \%$ of cases. Motor vehicle accidents (37\% motorcycle, $31 \%$ automobile and $7 \%$ run over) predominated. Fall from height caused $18 \%$ of fractures.

Fifty-two percent of cases were open wound fractures. Gustillo and Anderson grade IIIA was predominant. Only one case developed skin coverage failure (Gustillo and Anderson grade IIIB), which was covered with a local gastrocnemius muscle flap. Soft tissue conditions determined time of definitie osteosynthesis with internal fixator. Patients with severe soft tissue damage underwent staged treatment, initiated by external fixation and followed by use of an internal fixator. All open fractures underwent staged treatment, with temporary transarticular external fixator. Among closed fractures, $71 \%$ received definitive treatment with LISS ${ }^{\circledR}$ plate.

Mean time elapsed from trauma to definitive fixation was 7.8 days (range 2-21). Mean hospital stay was 17.2 days (range 3-33). Thirty-four percent of patients underwent physical therapy during hospitalization with CPM (Continuous Pas- sive Motion) machine. The rate of bone healing was $93 \%$ at a mean time of 5.5 months (range 3-19). Primary consolidation without bone grafting occurred in 25 fractures (86\%). Two fractures (7\%) healed after bone graft at a second time, and two (7\%) showed no consolidation with this fixation method. A case, which evolved to pseudarthrosis, initially presented $8 \mathrm{~cm}$ bone defect. It underwent two attempts of bone grafting. Nevertheless, it has evolved with nonunion and breakage of distal screws of the implant. There was resolution after new osteosynthesis with absolute stability technique and replacement of the implant by a DCS ${ }^{\circledR}$ (Dynamic Condylar Screw, Synthes) plate. Another case underwent bone grafting with internal fixation after 10 months of fracture, and is currently at two months postoperatively awaiting consolidation, showing good evolution. In the radiographic evaluation of patients we identified that malalignment of the femur was caused by two factors: firstly because of failed initial reduction, which is related to the difficulty of implant placement. The other factor regards the angular loss during patient follow-up, due to failure implant fixation. Eight patients (27.5\%) had malalignment of the anatomical axis of the femur, all due to failure of initial reduction, intra-operatively, seven in the coronal plane and one in the sagittal plane. One of these cases had additional angular loss during follow-up. This one, which initially had been reduced and fixed in retrocurvatum, suffered plate break on at its 
distal region at five months after surgery, evolving with varus malunion. (Figure 1)

There were no cases of infection. There were two proximal loosening of the implant, requiring resetting with the same material followed by a good evolution. In one patient implant was removed due to a complaint of knee pain. Three patients developed knee stiffness (flexion $<90^{\circ}$ ), two cases were resolved with joint manipulation under anesthesia and one refused to undergo subsequent surgical procedures. (Table 1)

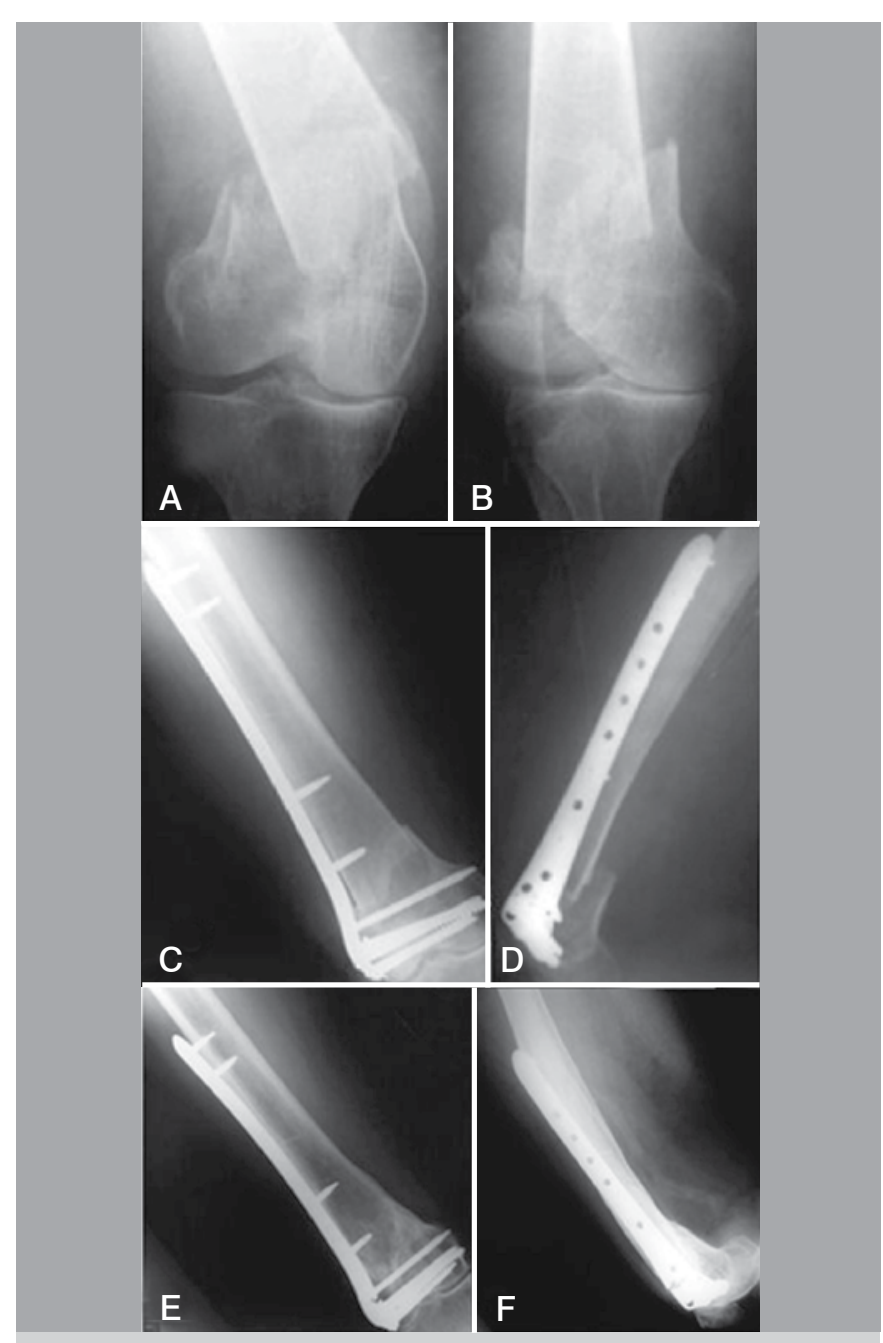

Figure 1. Seventy-seven year old female patient, who fell from height, right side fracture $\mathrm{AO}$ type 33-C2: ( $\mathrm{A}$ and $\mathrm{B}$ ) $\mathrm{X}$-ray on day of trauma. ( $\mathrm{C}$ and $\mathrm{D}$ ) Immediate post-operative $X$-rays showing failure of initial reduction. ( $E$ and F) X-ray at follow up, one year post-operative, showing breakage of plates at their distal region, consolidated fracture and varus angulation.

\section{DISCUSSION}

There are several options for management of distal femur fractures. In the last decade, the use of LISS $^{\circledR}$ type internal fixation brought a new perspective for the treatment of articular fractures cases with small distal fragment and fractures in osteoporotic bones. ${ }^{7}$ Recent biomechanical studies, such as Higgins et al. ${ }^{14}$ demonstrated benefits of plates with locking screws compared to conventional plates in cyclic stress assays and axial loading tests. Duffy et al..$^{15}$ in their model study of AO 33-C3 type fractures measured plastic deformity after cyclic assays, and elastic after
Table 1. Post-operative complications.

\begin{tabular}{c|c}
\hline Infection & 0 \\
\hline Pseudarthrosis & $2(7 \%)$ \\
\hline Nonunion & $2(7 \%)$ \\
\hline Implant loosening & $2(7 \%)$ \\
\hline Implant breakage & $2(7 \%)$ \\
\hline Fail at initial reduction & $8(27.5 \%)$ \\
\hline Coronal plane & $7(24 \%)$ \\
\hline Sagittal plane & $1(3.5 \%)$ \\
\hline Angular loss of coronal plane & $1(3.5 \%)$ \\
\hline Angular loss of sagittal plane & 0 \\
\hline Deviation of final anatomical axis & $8(27.5 \%)$ \\
\hline Intraarticular screw & 0 \\
\hline Stiffness of the knee & $3(10.3 \%)$ \\
\hline
\end{tabular}

static charges. These authors showed a higher strength and flexibility of LISS ${ }^{\circledR}$ plate in relation to conventional plates. Kregor et al. ${ }^{16}$, in a clinical study, presented a series of 123 distal femur fractures treated with LISS ${ }^{\circledR}$ plate, obtaining $93 \%$ of primary bone consolidation. Five cases of proximal implant loosening, two cases of pseudarthrosis and three acute infections were observed. There was no loss in varus angulation. Malalignment was observed in $6 \%$ of cases.

Schutz et al. ${ }^{17}$, in a series of 62 patients reported primary bone healing in $85 \%$ of patients, with a malalignment of the femur in the coronal plane in $26 \%$ of patients, and in sagittal plane in $15 \%$ of cases. The authors reported a $3 \%$ infection rate.

Weight et al. ${ }^{18}$ reported $100 \%$ primary bone healing without other procedures, with three cases (13\%) of malalignment, no infection and with good final range of motion of the knee.

The present study has limitations related to its retrospective nature, which aims to describe the experience of our institution in the indication and treatment of fractures of the distal femur with the technique described.

Our data are consistent with reports from most authors: healing rate between $85 \%$ to $100 \%$ of cases. There was a rate of bone grafting $(14 \%)$ similar to those shown in the literature $(0-15 \%){ }^{16-18}$ We had a similar rate of implant breakage (7\%). Nonconsolidation rate was $7 \%$. The rate of infection and implant loosening was similar to the literature. In our series $27.5 \%$ of patients evolved with malalignment in the frontal plane.

Up to $26 \%$ of patients treated with LISS plate ${ }^{\circledR}$ also evolved to axis deviation as reported by several autores. ${ }^{16-18}$ Proper placement of the implant associated with a good reduction was a difficulty. Twenty-seven percent of the cases had failed in the reduction initially, with axis deviation in the immediate postoperative period. This finding is probably due to the difficulty of controlling the reduction in cases of failures in metaphyseal comminuted fractures, besides the difficulty in adapting an implant sized to a population ethnically very distinct from ours. Another major factor is that differently from ordinary boards, on internal fixation the use of head-threaded screws through the plate does not help fracture reduction. Thus, before fixing, 
surgeon must ensure reduction in the coronal plane, sagittal plane and rotation are satisfactory.

The reduction in the sagittal plane is facilitated by the plate mould, which is specific to the third distal region of the femur, guiding the surgeon refarding the alignment of the epiphyseal fragment in lateral view. This does not occur in the coronal plane. An additional difficulty was that in most cases of our sample the fractures presented with complex metaphyseal and articular comminution (41\% AO type 33-C3).

\section{CONCLUSION}

New implants, despite of their biomechanical advantages measured in the laboratory, does not exclude the basic principles of joint anatomical reduction and restoration of mechanical and rotational axis of the limb. These tasks depend on the surgeon, who is responsible for evaluating the quality of intraoperative reduction. The prognosis of knee function is directly related to surgical technique accuracy.

\section{REFERENCES}

1. Arneson TJ, Melton LJ 3rd, Lewallen DG, O'Fallon WM. Epidemiology of diaphyseal and distal femoral fractures in Rochester, Minnesota, 1965-1984. Clin Orthop Relat Res. 1988;(234):188-94

2. Zlowodzki M, Bhandari M, Marek DJ, Cole PA, Kregor PJ. Operative treatment of acute distal femur fractures: systematic review of 2 comparative studies and 45 case series (1989 to 2005). J Orthop Trauma. 2006;20(5):366-71.

3. Neer CS 2nd, Grantham SA, Shelton ML. Supracondylar fracture of the adult femur. A study of one hundred and ten cases. J Bone Joint Surg Am. 1967;49(4):591-613.

4. Stewart MJ, Sisk TD, Wallace SL. Fractures of the distal third of the femur. J Bone Joint Surg Am. 1966;48:784-807.

5. Schatzker J, Lambert DC. Supracondylar fractures of the femur. Clin Orthop Relat Res. 1979;(138):77-83.

6. Stover M. Distal femoral fractures: Current treatment, results and problems. Injury. 2001;32(Suppl 3):SC3-13.

7. Higgins TF. Distal femoral fractures. J Knee Surg. 2007;20(1):56-66

8. Schatzker J, Mahomed N, Schiffman K, Kellam J. Dynamic Condylar screw: a new device. A preliminary report. J Orthop Trauma. 1989;3(2):124-32.

9. Krettek C, Müller M, Miclau T. Evolution of minimally invasive plate osteosynthesis (MIPO) in the femur. Injury. 2001;32(Suppl 3):SC14-23.

10. Farouk O, Krettek C, Miclau T, Schandelmaier P, Guy P, Tscherne H. Minimally invasive plate osteosynthesis: does percutaneous plating disrupt femoral blood supply less than the traditional technique? J Orthop Trauma. 1999;13(6):401-6.

11. Frigg R, Appenzeller A, Christensen R, Frenk A, Gilbert S, Schavan R. The development of the distal femur Less Invasive Stabilization System (LISS). Injury. 2001;32(Suppl 3):SC24-31.

12. Zlowodzki M, Williamson S, Cole PA, Zardiackas LD, Kregor PJ. Biomechanica evaluation of the less invasive stabilization system, angled blade plate, and retrograde intramedullary nail for the internal fixation of distal femur fractures. J Orthop Trauma. 2004;18(8):494-502.

13. Morelans JR, Basset LW, Hanker GJ. Radiographic analysis of the axial alignment of the lower extremity. J Bone Joint Surg Am. 1987;69(5):745-9.

14. Higgins TF, Pittman G, Hines J, Bachus KN. Biomechanical analysis of distal femur fracture fixation: fixed-angle screw-plate construct versus condylar blade plate. J Orthop Trauma. 2007;21(1):43-6.

15. Duffy P, Trask K, Hennigar A, Barron L, Leighton RK, Dunbar MJ. Assessment of fragment micromotion in distal femur fracture fixation with RSA. Clin Orthop Relat Res. 2006;448:105-13.

16. Kregor PJ, Stannard JA, Zlowodzki M, Cole PA. Treatment of distal femur fractures using the less invasive stabilization system: surgical experience and early clinical results in 103 fractures. J Orthop Trauma. 2004;18(8):509-20.

17. Schutz M, Muller M, Regazzoni P, Hontzsch D, Krettek C, Van der Werken C et al. Use of the less invasive stabilization system (LISS) in patients with distal femoral (AO33) fractures: a prospective multicenter study. Arch Orthop Trauma Surg. 2005;125(2):102-8.

18. Weight $\mathrm{M}$, Collinge $\mathrm{C}$. Early results of the less invasive stabilization system for mechanically unstable fractures of the distal femur (AO/OTA types A2, A3, C2, and C3). J Orthop Trauma. 2004;18(8):503-8. 